\title{
LA REPRESENTACIÓN DEL SUICIDIO EN LA PRENSA ESPAÑOLA
}

\section{THE REPRESENTATION OF SUICIDE IN THE SPANISH PRESS}

\author{
María Teresa Santos Diez \\ Idoia Camacho Markina*
}

\section{RESUMEN}

En este artículo se analiza el tratamiento al tema del suicidio en los diarios españoles $L a$ Razón, El Mundo, El País y ABC desde el 1 de enero al 31 de diciembre de 2017. Mediante la metodología de análisis de contenido, se cuantifica la autoría de los textos, los géneros $y$ las fuentes empleadas, además se identifican las áreas temáticas que centran el interés de los diarios analizados. Las conclusiones indican que las noticias son fundamentalmente descriptivas y con poca especialización con textos que priman los aspectos negativos e, incluso, sensacionalistas. Los temas principales se centran en el suicidio consumado, la cultura y la tentativa de suicidio. Asimismo, se presenta el suicidio como una respuesta o una solución para enfrentarse a los problemas.

PALABRAS CLAVE: ESPAÑA * SUICIDIO * PRENSA * PREVENCIÓN * MOTIVOS

\section{ABSTRACT}

This article analyzes the treatment that the Spanish newspapers La Razón, El Mundo, El País and $A B C$ gave to the subject of suicide from the 1st of January to the 31st of December of 2017. Through the methodology of content analysis, we have quantified the authorship of the texts, the genres and the information sources used. The conclusions indicate that news are basically descriptive and with little specialization and texts that dominate the negative and even sensational aspects. The main topics are consummated suicide, culture and attempted suicide. Suicide is also presented as a response or solution at the moment of facing problems.

KEYWORDS: SPAIN * SUICIDE * PRESS * PREVENTION * MOTIVES

Universidad del País Vasco/Euskal Herriko Unibertsitatea, España.

mariateresa.santos@ehu.eus

** Universidad del País Vasco/Euskal Herriko Unibertsitatea, España.

idoia.camacho@ehu.eus 


\section{1) INTRODUCCIÓN}

Etimológicamente, la palabra "suicidio" procede del latín sui (sí mismo) y caedere (matar). El suicidio constituye un hecho humano transcultural $y$ universal, presente en todas las épocas desde el origen de la humanidad (Mansilla, 2010). No es un acto fortuito, sino que se trata de un problema complejo motivado por causas difíciles de descubrir, en el que resultan determinantes factores psicológicos, sociales, biológicos, culturales y ambientales (Ruíz y Olry, 2006; Hawton y van Heeringen, 2009). La Organización Mundial de la Salud lo califica como un grave problema de salud pública a nivel mundial, el cual ha aumentado su incidencia en las últimas décadas, hasta el punto de que unas 800000 personas se quitan la vida cada año (Organización Mundial de la Salud, 2 de setiembre de 2019). Este dato eleva al suicidio a la segunda causa principal de defunción en el grupo de edad de 15 a 29 años, sin tener en cuenta que son muchas más las personas que no pasan del intento.

Según datos del Instituto Nacional de Estadísticas (19 de diciembre de 2018), en España, perdieron la vida 3679 personas por esta causa en el año 2017, lo que supone un 7,9 por 100 000 habitantes, de los cuales 2718 son hombres $(73,9 \%)$ y 961 mujeres $(26,1 \%)$, resultando la primera causa externa de muerte, por encima de los ahogamientos (6,7 por 100000 habitantes), las caídas accidentales $(6,6)$ y los accidentes de tráfico $(4,2)$. Aún con todo lo anterior, España posee una tasa de suicidios significativamente menor que la media europea $(15,4)$. En América Latina y el Caribe, la tasa era de 6,8 en 2016, aunque los datos son dispares entre los diferentes países (Banco Mundial, 2000-2016). En Costa Rica, la tasa ha ido aumentando desde un 7,3 en 2000, 7,9 en 2005, 8,6 en 2010 y 7,9 en 2015 y 2016. Los datos de 2016 son inferiores a países como Uruguay $(18,4)$, Cuba $(13,9)$, Bolivia y Nicaragua $(12,2)$, Haití $(11,7)$, Chile $(10,6)$, República Dominicana $(9,9)$, Paraguay $(9,5)$ y Argentina $(9,2)$, aunque superiores a otros como Colombia (7,2), Ecuador $(7,1)$, Brasil $(6,5)$, Perú $(4,9)$, Panamá $(4,3)$ o Venezuela $(3,7)$.

En lo que se refiere al tratamiento del suicidio en los medios de comunicación, la presente investigación parte de la base de que los medios no solo se dedican a informar, sino que construyen la realidad (Entman, 1993) e influyen en las opiniones $y$ actitudes de la sociedad (Wolf, 1994; Rodrigo, 1995; McQuail, 2000; Lippmann, 2003), modelando las actitudes, las creencias y los valores de las personas (Montero, 1993). Los acontecimientos que muestran los medios se presentan al público como auténticos, lo que hace creer que la realidad es tal y como aparece en los medios, que no hay opción alguna de actuar en otro sentido diferente al mostrado (Ramos, 1995). De esta manera, los medios modelan la percepción del mundo y hace partícipe al individup en su forma de entenderlo, determinando la imagen que se tiene de la realidad.

Este planteamiento se enmarca en la teoría de la construcción social de la realidad formulada por Berger y Luckmann (1972), que defiende que "la vida cotidiana se presenta como una realidad interpretada por los hombres y que para ellos tiene el significado subjetivo de un mundo coherente" (p. 36). Por tanto, la realidad es construida socialmente $y$, aunque estos autores no aluden en su obra a los medios periodísticos, se entiende que estos constituyen hoy el factor más importante de la construcción social de la realidad cotidiana, ya que "de sus orientaciones últimas dependerá el tipo de imagen de la realidad que acabará siendo conocida por el público" (Dader, 1990, p.23).

La influencia de los medios de comunicación en la construcción de la realidad ha sido explicada por la teoría de la agenda-setting, la cual defiende que los medios de comunicación, al seleccionar y priorizar las informaciones que publican, dirigen la atención e influyen en la percepción de cuáles son los temas más importantes del día. Siguiendo a McCombs (2006), el público utiliza los mensajes de los medios para organizar su propia agenda y decidir cuáles son los temas sobre los que centrará su atención y su pensamiento, $y$ tal vez su acción, conviertiendo la agenda mediática en su agenda social.

Entre las consecuencias de los efectos de la fijación de la agenda, destaca la preparación previa sobre los puntos de vista que posteriormente guían la opinión de la sociedad, lo que 
lleva la influencia de los medios de comunicación como fijadores de la agenda al mismo centro del ruedo de la opinión pública (McCombs, 2006). Conocer la agenda de los medios resulta, pues, un factor clave para conocer los núcleos temáticos de interés social, las problemáticas sociales más relevantes y la mentalidad dominante en la ciudadanía sobre dichos temas $y$ problemáticas.

Sobre la base de esta teoría, numerosas investigaciones han analizado la influencia de los medios de comunicación sobre los índices de suicidios. Phillips (1974) acuñó el término "efecto Werther" para describir un aumento en las conductas suicidas inmediatamente después de la publicación de noticias relacionadas con el suicidio en los periódicos. El fundamento teórico del efecto Werther es la teoría del aprendizaje social, que afirma que si la gente se entera de que hay personas que resuelven sus problemas mediante el suicidio, otros que se identifican con ellas pueden copiar el comportamiento (Muñoz y Sánchez, 2013).

En esta misma línea, en la literatura científica se encuentra una gran cantidad de estudios que respaldan la idea de que las noticias de los medios de comunicación y las tendencias suicidas están asociadas (Sisask y Värnik, 2012), de tal manera que un aumento en el espacio que dedican los medios al suicidio provoca una mayor cantidad de personas que se quitan la vida (Etzersdorfer y Sonneck, 1998; Pirkis y Warwick, 2001; Dare et al., 2011; Ladwig et al., 2012). Otra línea de investigación menos explorada pone en duda la prevalencia del efecto Werther y plantea que el problema no es tratar el suicidio en los medios, sino la forma de tratarlo. Es decir, si los medios informan correctamente sobre el tema pueden prevenirse las conductas suicidas, lo que se conoce como "efecto Papageno" (Etzersdorfer y Sonneck,1998; Niederkrotenthaler et al., 2010; Cheng et al., 2017), algo que ocurre tanto en los medios tradicionales como en los nuevos medios digitales (Ortiz y Khin, 2018).

Los resultados de estas investigaciones han llevado a la Organización Mundial de la Salud (OMS) a elaborar guías con directrices para la prevención del suicidio dirigidas a profesionales de los medios de comunicación, con el objetivo de que redacten las noticias sobre el suicidio de manera responsable (oms, 2008 ; 2017). Según estas guías, entre las prácticas periodísticas que se deben evitar es insistir en no tratar al suicidio de forma sensacionalista, ni glorificar a la persona que se suicida. Tampoco se debe presentar el suicidio como algo normal, como solución a los problemas o como un modo de afrontarlos.

Además, recomiendan no incluir la palabra suicidio en el titular, ni informar sobre el método empleado o el lugar del suicidio, evitar publicar fotografías o vídeos de la víctima en estado mortal, del método empleado, de la escena del suicidio o notas suicidas en ninguna de sus posibles modalidades, así como evitar cubrir de manera exagerada o sensacionalista suicidios de personas famosas. Por el contrario, algunas prácticas apropiadas son utilizar un lenguaje responsable $y$ entendible por todo el público, evitar simplificaciones excesivas, informar sobre el carácter prevenible de los suicidios, contribuir a reducir el estigma sobre trastornos mentales y mitos sobre el suicidio, educar al público acerca de cómo superar las conductas suicidas, respetar los sentimientos de los familiares y suministrar información sobre lugares a donde acudir para buscar ayuda.

En España se ha investigado poco sobre el tratamiento del suicidio en los medios. Olmo y García (2014) estudiaron el tratamiento del suicidio en TVE y en Canal Sur Televisión durante 2012 y determinaron que estos dos medios cubren el tema de manera escasa e inadecuada. Herrera et al. (2015) analizaron cinco diarios y concluyeron que la mayoría de sus noticias no tiene en cuenta las recomendaciones de la oms. Garrido, Eleazar y Catalán (2018) analizaron el diario $A B C$ durante los años 1995, 2000 y 2005, con lo cual determinaron que la cobertura ha mejorado a lo largo del tiempo, pero no en todos los aspectos recomendados por la OMS.

A pesar de la trascendencia social del suicidio, su tratamiento en los medios de comunicación es un tema poco investigado desde el ámbito académico de la Comunicación. El presente trabajo centra su objetivo en analizar 
el tratamiento que los diarios de información general españoles (El País, El Mundo, La Razón y $A B C$ ) han realizado sobre el suicidio durante el año 2017.

\section{2) OBJETIVOS E HIPÓTESIS}

Esta investigación plantea como objetivo general, el análisis del tratamiento que realiza la prensa diaria española sobre el suicidio. Se parte de los siguientes objetivos específicos:

1) Cuantificar las informaciones publicadas en cada diario sobre el suicidio.

2) Determinar las áreas temáticas que abordan los diarios.

3) Conocer si los diarios cumplen las recomendaciones de la oms sobre el tratamiento del suicidio en los medios.

Con el fin de conseguir los objetivos propuestos se parte de las siguientes hipótesis:

H1) El tema del suicidio forma parte de la agenda de los medios.

H2) Los textos publicados presentan un carácter informativo en detrimento de los géneros interpretativos y se elaboran con poca profundidad.

H3) Existe un predominio de noticias sobre el suicidio consumado.

H4) La mayor parte de la información publicada sobre suicidios no sigue las recomendaciones de la OMS.

\section{3) METODOLOGÍA}

Para realizar el estudio se han elegido las cabeceras de los diarios ${ }^{1}$ El País (1976), El Mundo (1989), ABC (1903) y La Razón (1998), atendiendo a criterios como calidad, cobertura, tirada y líneas editoriales diferentes que cubren todos los espacios sociológicos. El espacio temporal abarca desde 1 de enero hasta el 31 de diciembre de 2017. Como unidad de análisis se ha considerado cada una de las piezas publicada

1 Sus direcciones electrónicas son http://elpais.com; http://www.elmundo.es; http://www.abc.es; http:// www.larazon.es en los diarios con independencia del género periodístico, ya sea informativo, interpretativo o de opinión, siguiendo criterios establecidos por otros autores (Camacho, 2009; Marín, Armentia y Olábarri, 2016), que contenga el término "suicid" (Garrido et al., 2018, p. 812). Después de realizar la búsqueda desde los portales web de los diarios mencionados y descartar aquellas piezas que trataban el suicidio de manera tangencial, se han contabilizado 878 unidades que se analizaron en su totalidad: El País (n= 301, $34,3 \%), A B C(\mathrm{n}=296,33,6 \%)$, El Mundo $(\mathrm{n}=170$, 19,5\%) y La Razón $(\mathrm{n}=111,12,6 \%)$.

Para codificar los datos se elaboró una ficha específica con las siguientes variables: fecha de publicación, autoría, género periodístico, secciones, fuentes. En cuanto a la temática se ha tomado como referencia a Olmo y García (2014), Herrera et al. (2015) y Rátiva et al. (2013, p. 3), aunque se ha adecuado a las categorías de los textos publicados. Como método de estudio se utilizó el análisis de contenido por considerar su aplicación adecuada a este tipo de investigaciones. Autores de referencia académica como Berelson (1952) consideran que es una técnica de investigación objetiva y sistemática para el estudio cuantitativo del contenido manifiesto de la comunicación.

También facilita el estudio de los textos de una manera sistemática, objetiva $y$ cuantitativa (Wimmer y Dominick, 1996), y posibilita la subjetividad para encontrar lo oculto al investigador (Bardin, 2002). Para Lombard et al. (2002), el análisis de contenido resulta apropiado e, incluso, necesario para investigaciones de comunicación centradas en el análisis de los mensajes. Para comprobar la fiabilidad del proceso se utilizó el método de Holsti (1969). Se tomaron al azar 88 piezas (el 10\% del total) que fueron cifradas por separado por las dos investigadoras, obteniendo 85 y 84 coincidencias, lo que supone un nivel de acuerdo del 0,96\%. Autores como Landis y Koch (1977) e Igartua (2006) garantizan la fiabilidad de los resultados obtenidos, ya que el nivel de acuerdo mínimo que se debe alcanzar para que los datos sean fiables es de $0,80(80 \%)$. 


\section{4) RESULTADOS}

En los diarios analizados entre enero $y$ diciembre de 2017, se han encontrado 878 textos sobre el suicidio, repartidos del siguiente modo: El País 301 (34,3\%), ABC 296 (33,3\%), El Mundo 170 (19,5\%) y La Razón 111 (12,6\%). Los meses de julio $(10,1 \%)$, mayo $(11,1 \%)$ y septiembre $(10,4 \%)$ publican un mayor número de noticias; junio $(5,1 \%)$ y noviembre $(6,4 \%)$ han registrado menos información. No obstante, se producen discrepancias entre los diarios: El País dedica más atención en abril $(12,4 \%)$,
$A B C$ lo hace en septiembre (14,2\%), El Mundo en julio $(14,5 \%)$ y La Razón en mayo (18,9\%).

\subsection{GÉNEROS, AUTORÍA, FUENTES Y SECCIONES}

En lo que se refiere a los géneros periodísticos utilizados (gráfico 1), hay un predominio de los informativos con 764 piezas $(86,5 \%)$, frente a los de opinión (13,5\%). Destaca la noticia (702) en todos los diarios (El Mundo 90,5\%, La Razón 81,9\%, ABC 81,4\% y El País 71,8\%), seguida de la opinión (106 piezas) con diferencias entre El País (16\%) y $A B C(12,8 \%)$ y La Razón (12,7\%) y El Mundo (3,6\%).

\section{GRÁFICO 1 \\ TEMA DEL SUICIDIO SEGÚN GÉNEROS PERIÓDISTICO POR PERIÓDICO ESPAÑA, 2017}

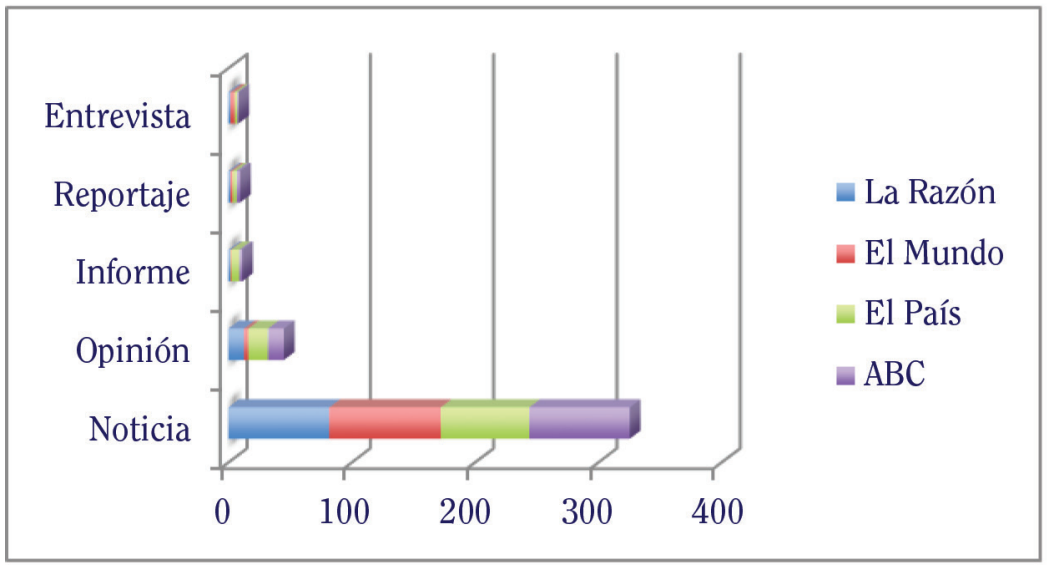

Fuente: Elaboración propia, 2017.

En cuanto a la autoría, en el gráfico 2 , destacan los textos firmados con el nombre de los redactores del periódico o colaboradores habituales con el 81,6\% (El País 80,7\%, La Razón 74,8\%, El Mundo 61,8\% y ABC 41,5\%). Son datos que revelan el interés por mostrar contenidos tratados de manera más objetiva $y$, en cierto modo, más especializada. Le siguen las piezas que proceden de agencia ( $A B C 32,8 \%$,
El Mundo 26,5\%, La Razón 8,1\% y El País 6,6\%) $y$ aquellos identificados con el nombre del diario (ABC 22,9\%, La Razón 13,5\%, El País 11,4\% y El Mundo 3,5\%). También se comprueba un escaso número de textos sin firma (La Razón 3,6\%, El Mundo 3,5\%, ABC 2,7\% y El País 1,3\%), informaciones que a menudo han sido elaboradas por agencias de noticias y se publican casi sin intervención de los periodistas (Gelado, 2009). 

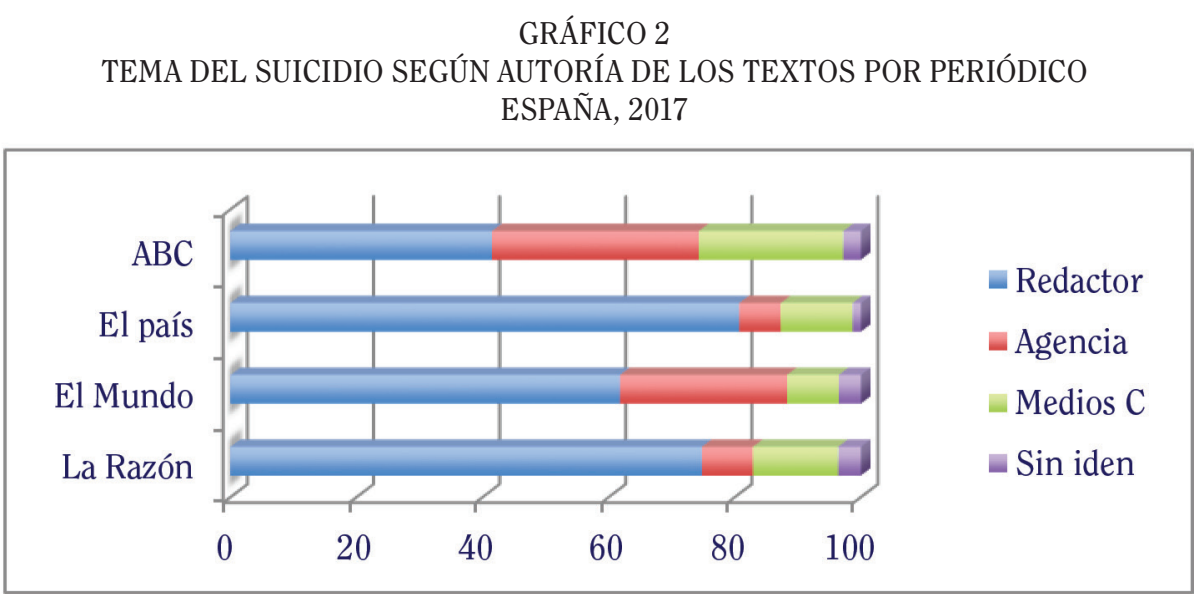

Fuente: Elaboración propia, 2017.

Las fuentes de información más utilizadas proceden de las instituciones (33,3\%), aunque con diferencias entre los diarios (El Mundo 38,2\%, El País 35,6\%, ABC 33\% y La Razón $22 \%$ ), le siguen las policiales $(30,2 \%)$ y científicas $(13,4 \%)$, aunque con discrepancia en los diarios, mientras que en
La Razón $(41,2 \%)$ y la $A B C(37,8 \%)$ predominan las policiales, en $E l$ Mundo $(38,2 \%)$ y El País $(35,6 \%)$ predominan las institucionales (tabla 1). Asimismo, en el 33,82 \% (297 piezas) de los casos no se determina la autoría (ABC 35,3, El País 26,5\%, El Mundo 19,1\% y La Razón 18,8\%).

TABLA 1

TIPOS DE FUENTES UTILIZADA

\begin{tabular}{|c|c|c|c|c|c|c|c|c|c|c|}
\hline \multirow{2}{*}{$\begin{array}{l}\text { FUENTES DE } \\
\text { INFORMACIÓN }\end{array}$} & \multicolumn{2}{|c|}{ LA RAZÓN } & \multicolumn{2}{|c|}{ EL MUNDO } & \multicolumn{2}{|c|}{ EL PAÍS } & \multicolumn{2}{|c|}{$\mathrm{ABC}$} & \multicolumn{2}{|c|}{ TOTAL } \\
\hline & NÚM. & $\%$ & NÚM. & $\%$ & NÚM. & $\%$ & NÚM. & $\%$ & NÚM. & $\%$ \\
\hline Institucionales & 23 & 22 & 62 & 38,27 & 117 & 35,67 & 75 & 33,03 & 277 & 33,35 \\
\hline Policiales & 45 & 41,28 & 52 & 32,09 & 67 & 20,42 & 86 & 37,88 & 250 & 30,26 \\
\hline Científicas & 14 & 12,84 & 12 & 7,40 & 54 & 16,46 & 31 & 13,65 & 111 & 13,43 \\
\hline Familiares & 7 & 6,42 & 9 & 5,55 & 36 & 10,97 & 15 & 6,60 & 67 & 8,11 \\
\hline Jurídicas & 11 & 10,09 & 13 & 8,02 & 12 & 3,65 & 9 & 3,96 & 45 & 5,44 \\
\hline $\begin{array}{l}\text { Medios de } \\
\text { comunicación }\end{array}$ & 8 & 7,33 & 10 & 6,17 & 17 & 5,18 & 6 & 2,7 & 41 & 4,96 \\
\hline Asociaciones & 1 & 0,09 & 4 & 2,46 & 25 & 7,62 & 5 & 2,64 & 35 & 4,23 \\
\hline TOTAL & 109 & 100 & 162 & 100 & 328 & 100 & 227 & 100 & 826 & 100 \\
\hline Textos sin fuente & 56 & 18,85 & 57 & 19,19 & 79 & 26,59 & 105 & 35,35 & 297 & 99,78 \\
\hline
\end{tabular}

Fuente: $\quad$ Elaboración propia, 2017. 
La mayor parte de los textos recogidos se ubican en la sección de sociedad con 182 piezas (La Razón 37,9\%, ABC 33,1\%, El Mundo 17\% y El País 4,4\%), seguida de internacional con 164 piezas (El País 24,6\%, ABC 16,4\%, La Razón 15,5\% y El Mundo 14,1\%,) y cultura con 135 piezas (El País 17,7\%, El Mundo 13,2\%, $A B C$ 14,8\% y La Razón 13,8\%). En cuanto a la procedencia de la información, destaca la estatal con el 60,1\% (ABC 64,9\%, El Mundo 61,2\%, La Razón 57,6\% y El País 55,8\%), dedicando mayor cobertura a Madrid (28,4\%) seguida de Cataluña (5,6\%) y Andalucía (5,1\%). En el ámbito internacional $(39,1 \%)$, se publica más información sobre suicidio en EE.UU (15,9\%), seguido de los países latinoamericanos (3,5\%).

\subsection{TEMÁTICA}

\section{SUICIDIO CONSUMADO}

Es el tema más destacado en todos los diarios, con un 39,5\% de los textos (347 piezas) (El País 42,1\%, La Razón 41,4\%, ABC 37,8\% y El Mundo 36,4\%). Le sigue cultura (El País 16,6\%, La Razón 15,3\%, ABC 9,1\% y El Mundo 7,6\%) y tentativa de suicidio (El Mundo 15,8\%, La Razón 13,5\%, El País 10,6\% y ABC 8,7\%). En menor medida aparecen temas como inducción al suicidio $(8,4 \%)$, legislación $(6,7 \%)$, prevención $(6,5 \%)$ y justicia/tribunales $(4,8 \%)$. En conjunto, las noticias sobre el suicidio consumado se publican en los meses de julio (20\%), diciembre $(10,9 \%)$ y septiembre $(9,2 \%)$; las de cultura en octubre (13,5\%), mayo (10,3\%), junio $y$ agosto $(8,4 \%$ cada mes); $y$ las de tentativa de suicidio en mayo (16\%), abril (11\%) y febrero (10\%). En cuanto a la procedencia geográfica, es más numerosa la estatal (59,9\%), que destacan Madrid (31,7\%) y Cataluña $(6,1 \%)$, que la internacional $(40,1 \%)$, EE.uU. $(17,1 \%) y$ países latinamericanos $(4,2 \%)$.

En esta temática se incluyen las noticias sobre suicidios de famosos (27\%). Se presenta la información con posibles factores determinantes al momento de consumar el acto (trastornos depresivos 18,4\%, violencia de género 12,2\% (42 piezas), datos similares a los obtenidos por Olmo y García (2014), tribunales 10,9\%, corrupción
$8 \%$ y acoso 7,9\%, entre otros) y en el 5,5 \% de los casos hay nota de suicidio, porcentaje que se incrementa hasta el $17,6 \%$ en Herrera et al (2015). En cuanto al mecanismo empleado, se especifica en el $62,5 \%$ de los casos (arma de fuego $20,2 \%$, ahorcamiento $16,4 \%$, precipitación $11,5 \%$, fármacos $6,6 \%$, sofocación $5,7 \%$ y explosión 2\%). Resultados que indican la misma tendencia de Garrido (2014), pero discrepan de los obtenidos por Baader et al. (2011) y Rátiva et al. (2013) en los que el ahorcamiento ocupa el primer lugar (76,3\% y 48\%).

Las piezas sobre suicidio consumado proceden del ámbito estatal (El Mundo 58,1\%, La Razón 50,9\%, El País 45,7\% y ABC 43,5\%). Cabe señalar que estos contenidos aparecen publicados durante todo el año, aunque registran mayor incidencia durante los meses de julio (20\%), diciembre (10,9\%) y septiembre $(9,2 \%)$. En cuanto al uso de las fuentes, destacan las institucionales en todos los diarios ( $E l$ Mundo 38,3\%, El País 35,7\%, ABC 33\% y La Razón 21,1\%). Algunos ejemplos de esta temática son: Un exempleado mata a cinco personas en una tienda de Orlando y se suicida (El País, 5/06/2017), Mueren un hombre y su hija al arrojarse por la ventana en el hospital La Paz (La Razón, 3/02/2017), Se confirma el suicidio del líder de Linkin Park: Chester Bennington (ABC, 22/07/2017) y Michael Mantenuto, actor de Disney, se suicida a los 35 años (El Mundo, 28/04/2017).

\section{CULTURA}

Apartado temático que agrupa las noticias que abordan el suicidio del mundo del cine, música, literatura, teatro, danza, arte y espectáculos en general $(12,1 \%)$, contenidos tratados de manera muy diferente en El País (16,6\%), La Razón (15,3\%), ABC (9,2\%) y El Mundo (7,6\%). Estos contenidos se han incrementado desde 13 (Olmo y García, 2014) hasta 107. Se trata de información que corresponde al ámbito estatal mayoritariamente $(73,9 \%)$ distribuida del siguiente modo: $76 \%$ en El País, $88,8 \%$ en $A B C, 15,4 \%$ en El Mundo y 88,3\% en La Razón. Su publicación se centra principalmente en los meses de abril $(12,5 \%)$, mayo $(11,5 \%)$ y septiembre $(10,3 \%)$. Predominan las fuentes procedentes de las propias 
redacciones $(52,3 \%)$, quedando relegadas a porcentajes muy escasos las institucionales $(2,8 \%)$ y las científicas (1,9\%). Algunos ejemplos de textos de esta temática son: Werther, amante suicida (El País, 12/05/2017), Jay Asher: Gente con instintos suicidas dice que mi libro les hizo pedir ayuda ( $A B C, 15 / 08 / 2017)$, Por 13 razones: la serie sobre el suicidio juvenil divide a los expertos ( $E l$ Mundo 11/05/2017), El suicidio de la razón (La Razón 25/12/2017).

\section{TENTATIVA DE SUICIDIO}

Los textos sobre tentativa de suicidio, es decir, el acto deliberado de quitarse la vida interrumpido antes de dar como resultado la muerte, suponen el 11,3\% de las piezas publicadas (El Mundo 15,8\%, La Razón 13,5\%, El País $10,6 \%$ y $A B C 8,7 \%)$. En su mayoría proceden del ámbito nacional (61\%), aunque con diferencias significativas entre los diarios (El Mundo 70,4\%, La Razón 66,6\%, ABC 57,6\% y El País $53,1 \%)$. Son textos que se publican mayoritariamente en los meses de mayo (16\%), abril (11\%) y febrero (10\%), aunque muestran diferencias: en El Mundo y La Razón predomina mayo $(22,2 \%$ y $33,3 \%)$, en $A B C$ el mes de septiembre (26,9\%) y en El País fue marzo (25\%).

Las fuentes más utilizadas proceden de instancias policiales (35\%), institucionales y sin determinar (20\% cada una), aunque los diarios muestran diferencias. Se destacan las policiales en La Razón (71,7\%) y en El País (37,4\%), mientras que en $A B C(26,5 \%)$ y en $E l$ Mundo son las institucionales $(31,7 \%)$. Sobre este tema se han publicado noticias como: Un hombre asesina a su pareja en Vicálvaro y después intenta suicidarse (El País, 3/03/2017), Evita el suicidio de su mujer sujetándola del pelo (La Razón, 7/02/2017), Deportan a un joven desde el CIE de Aluche tras varios intentos de suicidio ( $E l$ Mundo, 11/04/2017), La mayoría de los jóvenes con tentativa de suicidio confiesan que sufren soledad (ABC, 5/10/2017).

\section{INDUCCIÓN AL SUICIDIO}

La inducción al suicidio según el art. 143 del Código Penal (Ley Orgánica 10/1995, 23 de noviembre de 1995) supone que el suicida no hubiera tomado la fatal resolución de darse muerte si no existiera la conducta del inductor. Estos contenidos (8,4\%) tienen mayor presencia en $A B C(13,8 \%)$, que en La Razón (9\%), El País (6,6\%) y El Mundo (1,7\%). Corresponden al ámbito estatal (64,9\%), aunque con discrepancias en los diarios (La Razón 80\%, ABC 78\%, El País 70\% y El Mundo 25\%). En cuanto a los meses, en la $A B C$ predomina septiembre (17\%), en La Razón (33,3\%) y El País (40\%) el mes de mayo, y en El Mundo fueron los meses de julio, noviembre $y$ diciembre (33,3\% cada uno). Emplean como fuente principal las policiales $(16,3 \%)$ y las propias de la redacción $(14,9 \%)$.

Se han publicado noticias como: Condenada a 15 meses de cárcel la joven que animó a su novio mediante mensajes de texto a suicidarse (El País, 4/08/2017), Quince meses de cárcel para la joven que animó a su novio a suicidarse por SMS (La Razón, 4/08/2017), Retiran contenido de internet que incitaba al suicidio tras la denuncia del CAC (El Mundo, 19/05/2017) y Declaran culpable de homicidio a la joven que incitó a su novio a suicidarse a través de mensajes de texto (ABC, 16/06/2017).

\section{LEGISLACIÓN}

Esta temática recoge las noticias referentes a la legislación vigente, así como, los cambios legislativos referidos al suicidio, suicidio asistido y muerte digna que se producen en los distintos países. El diario que más textos publica es $A B C(9,7 \%)$, seguido de El Mundo (8,2\%) y El País (5,3\%). El 79,7\% proceden del ámbito estatal ( $A B C$ 86,2\%, El Mundo 78,6\% $y$ en El País 68,7\%). Las noticias sobre legislación se publican en marzo $(25,4 \%)$ y abril $(15,2 \%)$ y sus fuentes mayoritarias son institucionales $(71,2 \%)$. Se han publicado noticias como: Holanda, donde bien morir es cotidiano (El País, 3/09/2017), El Parlament votará una moción para legalizar la eutanasia y el suicidio asistido (El Mundo, 19/01/2017), Las 160 enmiendas a la ley de muerte digna empiezan a negociarse hoy en el Congreso ( $A B C$, 10/10/2017) y El Congreso tramita una ley de muerte digna (La Razón, 29/03/2017). 


\section{PREVENCION}

En esta temática se recogen las intervenciones $y$ las estrategias de prevención del suicidio desde el ámbito estatal, oms, privado y asociaciones, así como campañas de sensibilización, programas de prevención del suicidio adolescente, en personas en riesgo y para mejorar la imagen en los medios de comunicación. Supone el $6,5 \%$ de las piezas publicadas ( $E l$ Mundo 10\%, ABC 8,7\%, El País 3,3\% y La Razón 2,7\%). Al ámbito estatal pertenecen el 73,2\% de las piezas (La Razón 100\%, ABC 80,7\%, El Mundo 64,7\% y El País 60\%). Se publican más textos los meses de septiembre $(21,4 \%)$ y mayo $(14,7 \%)$, aunque con diferencias entre los diarios: $A B C$ publica en septiembre (30,8\%), La Razón (17,6\%) y El Mundo (33,3\%) en mayo, y El País en noviembre (30\%).

Sus fuentes son institucionales (34\%), científicas $(19,6 \%)$ y la propia redacción (14,9\%). Algunos ejemplos de esta temática son: Consejos para evitar el suicidio colectivo (El País, 16/03/2017), Facebook lanzará un nuevo sistema para prevenir el suicidio en sus usuarios (La Razón, 27/11/2017), El psicólogo examina hoy a Ignacio González para decidir si se le aplica el protocolo anti suicidio (El Mundo, 24/04/2017) y Combatir el suicidio desde la comprensión de 280 caracteres $(A B C$, 20/11/2017).

\section{JUSTICIA/TRIBUNALES}

Apartado que aborda sentencias judiciales que dictaminan si hay o no delito en los procesos judiciales y las denuncias relacionadas con el suicidio (4,7\%). Se distribuyen del siguiente modo: El Mundo (5,2\%), El País (4,8\%), ABC (4,7\%) y La Razón (3,6\%). La mayor parte de noticias pertenecen al ámbito estatal (54,8\%), aunque con diferencias (El Mundo 77,7\%, El País 53,3\%, ABC 50\% y La Razón 25\%). Las noticias sobre Justicia/Tribunales se publican los meses de enero (14,3\%), agosto $(14,3 \%)$ y diciembre $(11,9 \%)$. Sus fuentes proceden de las propias administraciones de justicia $(69,5 \%)$ y de las instituciones (19\%). Algunas noticias son: La juez ve indicios de acoso escolar a la menor que se suicidó en Murcia
(El País, 20/02/2017), Quince meses de cárcel para la joven que animó a su novio a suicidarse por SMS (La Razón, 4/08/2017), Se suicida un alto cargo militar de China investigado por corrupción (El Mundo, 28/11/2017), Denuncian ante la Fiscalía 15 contenidos de Internet que incitan al suicidio ( $A B C, 8 / 05 / 2017)$.

\section{TECNOLOGÍA/REDES SOCIALES}

En esta temática se incluyen contenidos relacionados con Internet, blogs y redes sociales como medios de difusión de contenidos que pueden ir desde la difusión de imágenes de suicidios en directo, hasta programas de detección del suicidio en los diarios analizados. Constituyen el 3,5\% de las piezas (El Mundo 8,8\%, El País 2,9\%, La Razón 2,7\% y ABC 1,3\%). El 70,9\% de los textos corresponde al ámbito nacional (El País 77,7\%, ABC 77,6\%, El Mundo 73,3\% y La Razón 33,3\%). Sus fuentes informativas son la propia redacción $(22,6 \%)$ y no se determinan en el 48,4\% de las piezas. Ejemplos de noticias son: Facebook desarrolla un algoritmo para detectar conductas suicidas antes que los humanos (El País, 27/11/2017), Suicidio 2.0: 16.700 «amigos» solo en un perfil de Twitter (La Razón, 9/05/2017), Críticas a Facebook por tardar dos semanas en eliminar el suicidio en directo de una niña de 12 años (El Mundo, 17/01/2017) y Facebook lanzará un nuevo sistema para prevenir suicidios entre sus usuarios (ABC, 29/11/2017).

\section{DIVULGATIVO}

Apartado que se centra en resultados de investigaciones y estudios relacionados con la conducta suicida, psicología, tratamientos $y$ recursos. Se han encontrado 30 piezas: $L a$ Razón (7,2\%), El País (3,3\%), ABC (2,7\%) y El Mundo (2,3\%). Datos similares a los obtenidos por Muñoz y Sanchez (2013). Las noticias de este tipo son de carácter (53,3\%), aunque con diferencias (El Mundo 100\%, ABC 62,5\%, El País 40\% y La Razón 37,5\%). En cuanto al uso de las fuentes, ocupan el primer lugar las procedentes del ámbito científico (80\%) seguido a mucha distancia de la redacción (10\%). Se ha publicado: Mil llamadas al día por acoso 
(ABC, 16/02/2017), El suicidio, un tabú social primera causa de muerte entre los jóvenes, a análisis en Bilbao (El Mundo, 4/11/2017), El mapa de la muerte digna en España (El País, 3/03/2017), El imposible mapa de los suicidios en España (El País, 14/06/2017).

\section{SUICIDIO ASISTIDO}

El Código Penal (art. 143.2) considera el suicidio asistido como la cooperación al suicidio de otra persona mediante actos positivos $y$ directos. Se trata de una temática escasamente abordada en los diarios (La Razón 3,6\%, ABC 2,3\%, El País 2,3\% y El Mundo 2,9\%). Proceden del ámbito estatal $(43,5 \%)$ y se publican sobre todo en septiembre (21,4\%), marzo y abril (21,9\% cada uno), aunque con diferencias entre los diarios analizado (ABC 42,8\%, El País 42,7\%, El Mundo 33,3\% y La Razón 50\%). Sus fuentes principales son la familia $(73,9 \%)$ y sin determinar (39,6\%). Algunas de estas noticias son: El suicidio asistido de Dj Fabo reabre el debate sobre la eutanasia en Italia (El País, 1/03/2017), El suicidio asistido supone el $4 \%$ del total de las muertes en Holanda (El Mundo, 14/04/2017), Un médico australiano presenta una máquina que te ayuda a morir en cinco minutos $(A B C$, $13 / 12 / 2017$ ) y El negocio de la muerte ( $L a$ Razón, 9/04/2017).

TABLA 2

TEMÁTICAS PRINCIPALES ABORDADAS CON RELACIÓN AL SUICIDIO SEGÚN PERIÓDICO ESPAÑA, 2017

\begin{tabular}{|c|c|c|c|c|c|c|c|c|c|c|}
\hline \multirow{2}{*}{ TEMÁTICAS } & \multicolumn{2}{|c|}{ La Razón } & \multicolumn{2}{|c|}{ El Mundo } & \multicolumn{2}{|c|}{ El País } & \multicolumn{2}{|c|}{$\mathrm{ABC}$} & \multicolumn{2}{|c|}{ TOTAL } \\
\hline & NÚM. & $\%$ & NÚM. & $\%$ & NÚM. & $\%$ & NÚM. & $\%$ & NÚM. & $\%$ \\
\hline Suicidio & 46 & 41,44 & 62 & 36,47 & 127 & 42,19 & 112 & 37,83 & 347 & 39,52 \\
\hline Cultura & 17 & 15,31 & 13 & 7,64 & 50 & 16,61 & 27 & 9,12 & 107 & 12,18 \\
\hline Tentativa s. & 15 & 13,51 & 27 & 15,88 & 32 & 10,63 & 26 & 8,78 & 100 & 11,38 \\
\hline Inducción s. & 10 & 9,00 & 3 & 1,76 & 20 & 6,64 & 41 & 13,85 & 74 & 8,42 \\
\hline Legislación & 0 & 0 & 14 & 8,23 & 16 & 5,31 & 29 & 9,79 & 59 & 6,71 \\
\hline Prevención & 3 & 2,70 & 17 & 10 & 10 & 3,32 & 26 & 8,78 & 56 & 6,5 \\
\hline $\begin{array}{l}\text { Justicia/ } \\
\text { Tribunales }\end{array}$ & 4 & 3,60 & 9 & 5,29 & 15 & 4,98 & 14 & 4,72 & 42 & 4,78 \\
\hline Tecnología & 3 & 2,7 & 15 & 8,82 & 9 & 2,99 & 4 & 1,35 & 31 & 3,53 \\
\hline Divulgativo & 8 & 7,20 & 4 & 2,35 & 10 & 3,32 & 8 & 2,70 & 30 & 3,41 \\
\hline S. asistido & 4 & 3,60 & 5 & 2,94 & 7 & 2,32 & 7 & 2,36 & 23 & 2,61 \\
\hline Terrorismo & 0 & 0 & 1 & 0,58 & 3 & 0,99 & 2 & 0,67 & 6 & 0,06 \\
\hline Otros & 1 & 0,09 & 0 & 0 & 2 & 0,66 & 0 & 0 & 3 & 0,03 \\
\hline TOTAL & 111 & 100 & 170 & 100 & 301 & 100 & 296 & 100 & 878 & 100 \\
\hline
\end{tabular}

Fuente: $\quad$ Elaboración propia, 2017.

\section{5) DISCUSIÓN Y CONCLUSIONES}

Este artículo tiene como objetivo el análisis del tratamiento que realiza la prensa diaria generalista española sobre el suicidio. $\mathrm{Su}$ interés radica en conocer la importancia que tiene el suicidio en la agenda diaria de los 
medios de comunicación y conocer si se trata de un tema relevante en la agenda mediática, por tanto, también en la social, tal como defiende McCombs (2006). Si se atiende a la teoría de la agenda setting, según la cual la realidad que perciben las personas es construida en gran parte por los medios de comunicación, el hecho de que los periódicos incluyan con cierta frecuencia en sus páginas noticias sobre suicidios conllevaría una mayor atención del público sobre este tema. Por otra parte, tal y como defienden las investigaciones que respaldan el efecto Werther y las que respaldan el efecto $\mathrm{Pa}$ pageno, si el tratamiento que hacen los diarios de esas noticias es correcto, se puede contribuir a la reducción de comportamientos suicidas en la sociedad, pero si es incorrecto, se puede contribuir a su aumento.

El estudio realizado demuestra que el suicidio es un tema recurrente en la prensa española, dado que en todos los periódicos analizados se publican textos durante todos los meses del año, aunque con predominio en los meses de julio, mayo y septiembre, fechas que coinciden con el suicidio de famosos (Herrera, et al., 2015). No obstante, se observan discrepancias en el número de textos publicados en cada periódico, así como, en la fecha de la publicación, por lo que no priman los mismos criterios. En lo que respecta a su ubicación, se obtienen resultados similares en los diarios analizados, que muestran un predominio de textos en las secciones sociedad internacional y cultura. En cuanto a la procedencia de las informaciones, destaca el ámbito estatal y comunidades autónomas frente a internacional, por lo que se puede considerar que prima la proximidad y cercanía (Herrera et al., 2015). De este modo, se confirma la primera hipótesis planteada (H1) y se concluye que la información sobre el suicidio sí forma parte de la agenda de los medios de comunicación.

El tratamiento que recibe el suicidio es eminentemente informativo. Se trata de noticias descriptivas redactadas con escasa profundidad y sin análisis, utilizando mayoritariamente una sola fuente de información, con un claro predominio de fuentes institucionales y policiales. Estos resultados confirman la segunda de las hipótesis establecidas (H2), es decir, son textos centrados en la actualidad con ausencia de especialización y escasa profundización periodística, algo que se puede relacionar con el hecho de que gran parte de ellos utilizan una sola fuente o no se determina la autoría. Además, las fuentes científicas, las familias y las asociaciones que trabajan el tema del suicidio - que son las que pueden aportar un contenido y visión más educativo sobre la prevención de las conductas suicidas - tienen poca presencia en las informaciones publicadas. Estos resultados se pueden interpretar como falta de compromiso de los diarios y escasa preocupación por la elaboración de las informaciones sobre este tema con un abordaje global.

La temática predominante es el suicidio como acto consumado, seguido de cultura, tentativa e inducción al suicidio y legislación. De este modo, se cumple la H3, que destacaba un predominio de noticias sobre el suicidio consumado. En general, se tiende a la simplificación de las noticias y no se abordan temas que se centren en aspectos que van más allá de los hechos acaecidos, como sería informar sobre la posibilidad de prevenir el suicidio o educar al público sobre cómo superar las conductas suicidas o dónde acudir en búsqueda de ayuda, tal y como recomienda la oms.

En los textos priman los aspectos negativos e, incluso, sensacionalistas, que se ponen de manifiesto en la inclusión de detalles como el mecanismo empleado, lo que también se destaca en otros estudios (Herrera et al. 2015; Rátiva et al. 2013; Muñoz y Sánchez, 2013; Baader et al. 2011; García y Barrantes, 2017; Garrido et al. 2018). Ofrecer información sobre el método empleado resulta contrario a las indicaciones de la oms, por considerar que pueden favorecer conductas suicidas (Sudak $y$ Sudak, 2005; Pirkis et al., 2006). También se han encontrado textos que ofrecen información sobre notas de suicidio, aunque en menor proporción que la encontrada en otros estudios (Acosta et al., 2017; Rátiva et al., 2013; Muñoz y Sánchez, 2013). Este tipo de información también se debe evitar publicar en cualquier modalidad, según la oms. 
Por otro lado, se ha observado que se presenta el suicidio como una respuesta o solución al momento de enfrentarse a problemas como la violencia de género, los tribunales, la corrupción y el acoso, entre otros. Si se atienden las indicaciones de la OMs, no se debe informar sobre el suicidio como el resultado de un solo factor, sino que es una causa muy compleja en la que intervienen factores como pueden ser psíquicos, físicos, personales, entre otros (Olmo y García, 2014). La H4 también queda demostrada y se concluye que los diarios analizados cumplen de manera escasa las recomendaciones de la OMS para la prevención del suicidio (Navarro-Gómez, 2017), aunque muestran una tendencia a la mejora con respecto a los estudios mencionados.

A pesar de ser un problema de salud pública de dimensión mundial, y que en España constituye primera causa externa de muerte no natural, los contenidos relacionados con la prevención de conductas suicidas, factores de riesgo o señales de alerta e informes divulgativos suponen el 6,5\% del total de textos publicados. Contenidos tan necesarios para visibilizar la conducta suicida y ayudar a levantar el tabú $y$ el estigma social que provoca el suicidio. Se constata la falta de información sobre las alternativas al suicidio, los recursos comunitarios, los factores de riesgo y las señales de alarma (Acosta et al., 2017; Olmo y García, 2014; Herrera et al., 2015).

Las investigaciones previas sobre el suicidio en los medios de comunicación han dejado en evidencia que estos pueden jugar un papel relevante para reducir los comportamientos suicidas, siempre que se informen de manera correcta y explicando aspectos sobre prevención $y$ servicios de ayuda. El presente estudio ratifica que, después de varias décadas, la prensa está aún lejos de cumplir las recomendaciones de la OMS y, por tanto, de llevar a cabo la importante función social mencionada que contribuiría a la disminución del número de suicidios.

\section{REFERENCIAS}

Acosta, F. J., Rodríguez, J. y Cejas, M. R. (2017). Noticias sobre suicidio en los medios de comunicación.
Recomendaciones de la oms. Revista Española de Salud Pública, 91.

Baader, T., Behne, P., Molina, J. L., Gacitúa, L., Yáñez, L., Urra, E. y Millán, R. (2011) ¿Está cambiando la prevalencia de los suicidios y sus características en la población chilena? Análisis de las tasas de suicidios y sus características sociodemográficas, ocurridas en la provincia de Valdivia, actual Región de Los Ríos, entre los años 1996 a 2008. Revista chilena de Neuro-Psiquiatría, 49(3), 273-282. doi: 10.4067/S0717-92272011000300008

Bardin, L. (2002). El análisis de contenido (3a. ed.). Akal Ediciones.

Berelson, B. (1952). Content analysis in communication researches. Free Press.

Berger, P. y Luckmann, T. (1972). La construcción social de la realidad. Amorrortu.

Camacho, I. (2009). La 'gripe A', en la prensa española. Revista Latina de Comunicación Social, 64, 827-843. doi: 10.4185/RLCS-64-2009-865-827-843

Cheng, Q., Chen, F., Lee, E. S. T. y Yip, P. S. F. (2017). The role of media in preventing student suicides: A Hong Kong experience. Journal of Affective Disorders, 227, 643-648. doi: 10.1016/j.jad.2017.11.007

Dader, J. L. (1990). Las provincias periodísticas de la realidad. Comunicación y Sociedad, 3 (1 y 2), 85-134.

Dare, A., Andriessen, K., Nordentoft, M., Meier, M., Huisman, A. y Pirkis, J. (2011). Media awards for responsible reporting of suicide: Experiences from Australia, Belgium and Denmark. International Journal of Mental Health Systems, 5, 5-15. doi: 10.1186/1752-4458-5-15

Entman, R. M. (1993). Framing: toward clarification of a fractured paradigm. Journal of Communication, 43(4), 51-58.

Etzersdorfer, E. y Sonneck, G. (1998). Preventing suicide by influencing mass-media reporting. The viennese experience 1980-1996. Archives of Suicide Research, 4(1), 67-74. doi: 10.1080/13811119808258290

García, J. D. y Barrantes, B. (2017). ¿Qué sabemos del suicidio de adultos en Costa 
Rica? Revista Pensamiento Actual, 17(28), 160-173.

Garrido-Fabián, F., Eleazar, A. y CatalánMatamoros, D. (2018). El uso por los periodistas de las recomendaciones de la oms para la prevención del suicidio. El caso del periódico Abc. Revista Latina de Comunicación Social, 73, 810-827. doi: 10.4185/RLCS-2018-1283

Gelado, R. (2009). La dependencia de la prensa española hacia las agencias de noticias. Comunicación y Sociedad, 22(2), 243-275.

Hawton, K. y van Heeringen, K. (2009). Suicide. Lancet (London, England), 373 (9672), 1372-81. doi: 10.1016/S01406736(09)60372-X

Herrera, R., Ures, B. y Martínez, J. J. (2015). El tratamiento del suicidio en la prensa española: ¿efecto werther o efecto papageno? Revista de la Asociación Española de Neuropsiquiatría, 35(125), 123-134. doi:10.4321/S0211-57352015000100009

Holsti, O. R. (1969). Content analysis for the social sciences and humanities. AddisonWesley.

Igartua, J. J. (2006). Métodos cuantitativos de investigación en comunicación. Bosch.

Ladwig, K. H., Kunrath, S., Lukaschek, K. y Baumert, J. (2012). The railway suicide death of a famous German football player: Impact on the subsequent frequency of railway suicide acts in Germany. Journal of Affective Disorders, 136 (1-2), 194-198. doi: 10.1016/j.jad.2011.09.044

Landis, J. R. y Koch, G. G. (1977). The measurement of observer agreement for categorical data. Biometerics, 33(1), 159-174.

Ley Orgánica 10/1995. (23 de noviembre de 1995). Código Penal. BoE, 281, de 24/11/1995.

Lippmann, W. (2003). La opinión pública. Langre.

Lombard, M., Snyder-Duch, J. y Bracken, C. C. (2002). Content analysis in mass communication: Assessment and reporting of intercoder reliability. Human Communication Research, 28(4), 587604. doi: 10.1111/j.1468-2958.2002. tb00826.x
Mansilla, F. (2010). Suicidio y prevención. Intersalud.

Marín, F., Armentia, J. y Olabarri, E. (2016). Alimentación y Salud: Enfoques predominantes en prensa española. Revista Latina de Comunicación Social, 71, 632 -653. doi: 10.4185/RLCS-2016-1113

McCombs, M. (2006). Estableciendo la agenda: El impacto de los medios en la opinión pública y en el conocimiento. Paidós.

McQuail, D. (2000). Introducción a la teoría de la comunicación de masas. Paidós.

Montero, M. D. (1993). La información periodística y su influencia social. Labor.

Muñoz, L. F. y Sánchez, R. (2013). Caracterización de noticias sobre suicidio en medios impresos en Colombia. Revista Colombiana de Psiquiatría, 42(1), 12-18.

Navarro-Gómez, N. (2017). El suicidio en jóvenes en España: Cifras y posibles causas. Análisis de los últimos datos disponibles. Clínica y Salud, 28(1), 25-31. doi:10.1016/j.clysa.2016.11.002

Niederkrotenthaler, T., Voracek, M., Herberth, A., Till, B., Strauss, M., Etzersdorfer, E., Eisenwort, B. y Sonneck, G. (2010). Role of media reports in completed and prevented suicide: werther $v$. papageno effects. The British Journal of Psychiatry, 197(3), 234-243. doi:10.1192/ bjp.bp.109.074633

Olmo, A. y García, D. (2014). El tratamiento de las noticias sobre suicidios. una aproximación a su reflejo en los medios de comunicación. Estudios sobre el Mensaje Periodístico, 20(2), 1149 . 1163. doi:10.5209/rev_ESMP.2014.v20. n2.47056

Organización Mundial de la Salud (2008). Preventing suicide: a resource for media professionals. http://www.who.int/mental_health/prevention/suicide/resource_media.pdf

Organización Mundial de la Salud (2017). Preventing suicide: a resource for media professionals. http://apps.who.int/iris/ bitstream/10665/258814/1/WHO-MSDMER-17.5-eng.pdf?ua=1 
Ortiz, P. y Khin, E. (2018). Traditional and new media's influence on suicidal behavior and contagion. Behavioral Sciences $y$ the Law, 36(2), 245-256. doi: 10.1002/ bsl.2338

Phillips, D. P. (1974). The influence of suggestion on suicide: Substantive and theoretical implications of the werther effect. American Sociological Review, 39(3), 340-354. doi:10.2307/2094294

Pirkis, J. y Warwick, B. R. (2001). Suicide and the media. part I: Reportage in nonfictional media. Crísis, 22 (4), 146-54.

Pirkis, J. y Warwick, B. R., Beautrais, A., Burgess, P. y Skehan, J. (2006). Media guidelines on the reporting of suicide. Crisis: The Journal of Crisis Intervention and Suicide Prevention, 27(2), 82-87.

Ramos, C. (1995). Los medios de comunicación, constructores de lo real. Comunicar, 5(3), 108-112. doi: https://doi.org/10.3916/ C05-1995-20

Rátiva, J. S., Ruíz, V. A. y Medina, O. A. (2013). Análisis de las noticias sobre suicidio publicadas en un diario local del Quindío, Colombia, entre 2004 y 2011. Revista Cubana de Higiene y Epidemiologia, 51(2), 184-191.

Rodrigo, M. (1995). Los modelos de la comunicación. Tecnos.

Ruíz, I. y Olry, A. (2006). El suicidio en la España de hoy. Gaceta Sanitaria, 20(1), 25-31. doi: https://doi. org/10.1157/13086023

Sisask, M. y Värnik, A. (2012). Media roles in suicide prevention: A systematic review. International Journal of Environmental Research and Public Health, 9(1), 12338. doi: 10.3390/ijerph9010123

Sudak, H. S. y Sudak, D. M. (2005). The media and suicide. Academic Psychiatry, 29(5), 495-499.

Wimmer, R. y Dominick, J. (1996). Mass media research. An introduction. Bosch

Wolf, M. (1994). Los efectos sociales de los media. Paidós.

Fecha de ingreso: 09/04/2019 Fecha de aprobación: 23/06/2020 